\title{
Hermite and poly-Bernoulli mixed-type polynomials
}

Dae San Kim ${ }^{1}$ and Taekyun Kim²

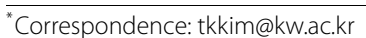
${ }^{2}$ Department of Mathematics, Kwangwoon University, Seoul, 139-701, Republic of Korea Full list of author information is available at the end of the article

\section{Abstract}

In this paper, we consider Hermite and poly-Bernoulli mixed-type polynomials and investigate the properties of those polynomials which are derived from umbral calculus. Finally, we give various identities associated with Stirling numbers, Bernoulli and Frobenius-Euler polynomials of higher order.

\section{Introduction}

For $r \in \mathbb{Z}_{\geq 0}$, as is well known, the Bernoulli polynomials of order $r$ are defined by the generating function to be

$$
\sum_{n=0}^{\infty} \frac{\mathbb{B}_{n}^{(r)}(x)}{n !} t^{n}=\left(\frac{t}{e^{t}-1}\right)^{r} e^{x t} \quad(\text { see }[1-16])
$$

For $k \in \mathbb{Z}$, the polylogarithm is defined by

$$
\operatorname{Li}_{k}(x)=\sum_{n=1}^{\infty} \frac{x^{n}}{n^{k}}
$$

Note that $\operatorname{Li}_{1}(x)=-\log (1-x)$.

The poly-Bernoulli polynomials are defined by the generating function to be

$$
\frac{\operatorname{Li}_{k}\left(1-e^{-t}\right)}{1-e^{-t}} e^{x t}=\sum_{n=0}^{\infty} B_{n}^{(k)}(x) \frac{t^{n}}{n !} \quad(\text { see }[5,8])
$$

When $x=0, B_{n}^{(k)}=B_{n}^{(k)}(0)$ are called the poly-Bernoulli numbers (of index $k$ ).

For $v(\neq 0) \in \mathbb{R}$, the Hermite polynomials of order $v$ are given by the generating function to be

$$
e^{-\frac{v t^{2}}{2}} e^{x t}=\sum_{n=0}^{\infty} H_{n}^{(v)}(x) \frac{t^{n}}{n !} \quad(\text { see }[6,12,13])
$$

When $x=0, H_{n}^{(v)}=H_{n}^{(v)}(0)$ are called the Hermite numbers of order $v$. 
In this paper, we consider the Hermite and poly-Bernoulli mixed-type polynomials $H B_{n}^{(v, k)}(x)$ which are defined by the generating function to be

$$
e^{-\frac{v t^{2}}{2}} \frac{\operatorname{Li}_{k}\left(1-e^{-t}\right)}{1-e^{-t}} e^{x t}=\sum_{n=0}^{\infty} H B_{n}^{(v, k)}(x) \frac{t^{n}}{n !}
$$

where $k \in \mathbb{Z}$ and $v(\neq 0) \in \mathbb{R}$.

When $x=0, H B_{n}^{(\nu, k)}=H B_{n}^{(v, k)}(0)$ are called the Hermite and poly-Bernoulli mixed-type numbers.

Let $\mathcal{F}$ be the set of all formal power series in the variable $t$ over $\mathbb{C}$ as follows:

$$
\mathcal{F}=\left\{f(t)=\sum_{k=0}^{\infty} a_{k} \frac{t^{k}}{k !} \mid a_{k} \in \mathbb{C}\right\} .
$$

Let $\mathbb{P}=\mathbb{C}[x]$ and $\mathbb{P}^{*}$ denote the vector space of all linear functionals on $\mathbb{P}$.

$\langle L \mid p(x)\rangle$ denotes the action of the linear functional $L$ on the polynomial $p(x)$, and we recall that the vector space operations on $\mathbb{P}^{*}$ are defined by $\langle L+M \mid p(x)\rangle=\langle L \mid p(x)\rangle+\langle M \mid p(x)\rangle$, $\langle c L \mid p(x)\rangle=c\langle L \mid p(x)\rangle$, where $c$ is a complex constant in $\mathbb{C}$. For $f(t) \in \mathcal{F}$, let us define the linear functional on $\mathbb{P}$ by setting

$$
\left\langle f(t) \mid x^{n}\right\rangle=a_{n} \quad(n \geq 0) .
$$

Then, by (1.6) and (1.7), we get

$$
\left\langle t^{k} \mid x^{n}\right\rangle=n ! \delta_{n, k} \quad(n, k \geq 0)
$$

where $\delta_{n, k}$ is the Kronecker symbol.

For $f_{L}(t)=\sum_{k=0}^{\infty} \frac{\left\langle L \mid x^{k}\right\rangle}{k !} t^{k}$, we have $\left\langle f_{L}(t) \mid x^{n}\right\rangle=\left\langle L \mid x^{n}\right\rangle$. That is, $L=f_{L}(t)$. The map $L \mapsto f_{L}(t)$ is a vector space isomorphism from $\mathbb{P}^{*}$ onto $\mathcal{F}$. Henceforth, $\mathcal{F}$ denotes both the algebra of formal power series in $t$ and the vector space of all linear functionals on $\mathbb{P}$, and so an element $f(t)$ of $\mathcal{F}$ will be thought of as both a formal power series and a linear functional. We call $\mathcal{F}$ the umbral algebra and the umbral calculus is the study of umbral algebra. The order $O(f)$ of the power series $f(t) \neq 0$ is the smallest integer for which $a_{k}$ does not vanish. If $O(f)=0$, then $f(t)$ is called an invertible series. If $O(f)=1$, then $f(t)$ is called a delta series. For $f(t), g(t) \in \mathcal{F}$, we have

$$
\langle f(t) g(t) \mid p(x)\rangle=\langle f(t) \mid g(t) p(x)\rangle=\langle g(t) \mid f(t) p(x)\rangle .
$$

Let $f(t) \in \mathcal{F}$ and $p(x) \in \mathbb{P}$. Then we have

$$
f(t)=\sum_{k=0}^{\infty} \frac{\left\langle f(t) \mid x^{k}\right\rangle}{k !} t^{k}, \quad p(x)=\sum_{k=0}^{\infty} \frac{\left\langle t^{k} \mid p(x)\right\rangle}{k !} x^{k} \quad(\text { see }[8,9,11,13,14]) .
$$

By (1.10), we get

$$
p^{(k)}(0)=\left\langle t^{k} \mid p(x)\right\rangle=\left\langle 1 \mid p^{(k)}(x)\right\rangle,
$$

where $p^{(k)}(0)=\left.\frac{d^{k} p(x)}{d x^{k}}\right|_{x=0}$. 
From (1.11), we have

$$
t^{k} p(x)=p^{(k)}(x)=\frac{d^{k} p(x)}{d x^{k}} \quad(\text { see }[8,9,13])
$$

By (1.12), we easily get

$$
e^{y t} p(x)=p(x+y), \quad\left\langle e^{y t} \mid p(x)\right\rangle=p(y) .
$$

For $O(f(t))=1, O(g(t))=0$, there exists a unique sequence $s_{n}(x)$ of polynomials such that $\left\langle g(t) f(t)^{k} \mid x^{n}\right\rangle=n ! \delta_{n, k}(n, k \geq 0)$.

The sequence $s_{n}(x)$ is called the Sheffer sequence for $(g(t), f(t))$ which is denoted by $s_{n}(x) \sim(g(t), f(t))$.

Let $p(x) \in \mathbb{P}, f(t) \in \mathcal{F}$. Then we see that

$$
\langle f(t) \mid x p(x)\rangle=\left\langle\partial_{t} f(t) \mid p(x)\right\rangle=\left\langle\frac{d f(t)}{d t} \mid p(x)\right\rangle .
$$

For $s_{n}(x) \sim(g(t), f(t))$, we have the following equations:

$$
h(t)=\sum_{k=0}^{\infty} \frac{\left\langle h(t) \mid s_{k}(x)\right\rangle}{k !} g(t) f(t)^{k}, \quad p(x)=\sum_{k=0}^{\infty} \frac{\left\langle g(t) f(t)^{k} \mid p(x)\right\rangle}{k !} s_{k}(x),
$$

where $h(t) \in \mathcal{F}, p(x) \in \mathbb{P}$,

$$
\frac{1}{g(\bar{f}(t))} e^{y \bar{f}(t)}=\sum_{n=0}^{\infty} s_{n}(y) \frac{t^{n}}{n !}
$$

where $\bar{f}(t)$ is the compositional inverse for $f(t)$ with $f(\bar{f}(t))=t$,

$$
\begin{aligned}
& s_{n}(x+y)=\sum_{k=0}^{n}\left(\begin{array}{l}
n \\
k
\end{array}\right) s_{k}(y) p_{n-k}(x), \quad \text { where } p_{n}(x)=g(t) s_{n}(x), \\
& f(t) s_{n}(x)=n s_{n-1}(x), \quad s_{n+1}(x)=\left(x-\frac{g^{\prime}(t)}{g(t)}\right) \frac{1}{f^{\prime}(t)} s_{n}(x),
\end{aligned}
$$

and the conjugate representation is given by

$$
s_{n}(x)=\sum_{j=0}^{n} \frac{1}{j !}\left\langle g(\bar{f}(t))^{-1} \bar{f}(t)^{j} \mid x^{n}\right\rangle x^{j}
$$

For $s_{n}(x) \sim(g(t), f(t)), r_{n}(x) \sim(h(t), l(t))$, we have

$$
s_{n}(x)=\sum_{m=0}^{n} C_{n, m} r_{m}(x)
$$

where

$$
C_{n, m}=\frac{1}{m !}\left\langle\frac{h(\bar{f}(t))}{g(\bar{f}(t))} l(\bar{f}(t))^{m} \mid x^{n}\right\rangle \quad(\text { see }[8,9,13])
$$


In this paper, we consider Hermite and poly-Bernoulli mixed-type polynomials and investigate the properties of those polynomials which are derived from umbral calculus. Finally, we give various identities associated with Bernoulli and Frobenius-Euler polynomials of higher order.

\section{Hermite and poly-Bernoulli mixed-type polynomials}

From (1.5) and (1.16), we note that

$$
H B_{n}^{(v, k)}(x) \sim\left(e^{\frac{v t^{2}}{2}} \frac{1-e^{-t}}{\operatorname{Li}_{k}\left(1-e^{-t}\right)}, t\right)
$$

and, by (1.3), (1.4) and (1.16), we get

$$
\begin{aligned}
& B_{n}^{(k)}(x) \sim\left(\frac{1-e^{-t}}{\operatorname{Li}_{k}\left(1-e^{-t}\right)}, t\right), \\
& H_{n}^{(v)}(x) \sim\left(e^{\frac{v t^{2}}{2}}, t\right), \quad \text { where } n \geq 0 .
\end{aligned}
$$

From (1.18), (2.1), (2.2) and (2.3), we have

$$
t B_{n}^{(k)}(x)=n B_{n-1}^{(k)}(x), \quad t H_{n}^{(v)}(x)=n H_{n-1}^{(v)}(x), \quad t H B_{n}^{(v, k)}(x)=n H B_{n-1}^{(v, k)}(x) .
$$

By (1.5), (1.8) and (2.1), we get

$$
\begin{aligned}
H B_{n}^{(v, k)}(x) & =e^{-\frac{v t^{2}}{2}} \frac{\operatorname{Li}_{k}\left(1-e^{-t}\right)}{1-e^{-t}} x^{n}=e^{-\frac{v t^{2}}{2}} B_{n}^{(k)}(x) \\
& =\sum_{m=0}^{\left[\frac{n}{2}\right]} \frac{1}{m !}\left(-\frac{v}{2}\right)^{m}(n)_{2 m} B_{n-2 m}^{(k)}(x) \\
& =\sum_{m=0}^{\left[\frac{n}{2}\right]}\left(\begin{array}{c}
n \\
2 m
\end{array}\right) \frac{(2 m) !}{m !}\left(-\frac{v}{2}\right)^{m} B_{n-2 m}^{(k)}(x) .
\end{aligned}
$$

Therefore, by (2.5), we obtain the following proposition.

Proposition 1 For $n \geq 0$, we have

$$
H B_{n}^{(v, k)}(x)=\sum_{m=0}^{\left[\frac{n}{2}\right]}\left(\begin{array}{c}
n \\
2 m
\end{array}\right) \frac{(2 m) !}{m !}\left(-\frac{v}{2}\right)^{m} B_{n-2 m}^{(k)}(x) .
$$

From (1.5), we can also derive

$$
\begin{aligned}
H B_{n}^{(v, k)}(x) & =\frac{\operatorname{Li}_{k}\left(1-e^{-t}\right)}{1-e^{-t}} e^{-\frac{v t^{2}}{2}} x^{n}=\frac{\operatorname{Li}_{k}\left(1-e^{-t}\right)}{1-e^{-t}} H_{n}^{(v)}(x)=\sum_{m=0}^{\infty} \frac{\left(1-e^{-t}\right)^{m}}{(m+1)^{k}} H_{n}^{(v)}(x) \\
& =\sum_{m=0}^{n} \frac{1}{(m+1)^{k}} \sum_{j=0}^{m}\left(\begin{array}{c}
m \\
j
\end{array}\right)(-1)^{j} e^{-j t} H_{n}^{(v)}(x) \\
& =\sum_{m=0}^{n} \frac{1}{(m+1)^{k}} \sum_{j=0}^{m}\left(\begin{array}{c}
m \\
j
\end{array}\right)(-1)^{j} H_{n}^{(v)}(x-j) .
\end{aligned}
$$


Therefore, by (2.6), we obtain the following theorem.

Theorem 2 For $n \geq 0$, we have

$$
H B_{n}^{(v, k)}(x)=\sum_{m=0}^{n} \frac{1}{(m+1)^{k}} \sum_{j=0}^{m}\left(\begin{array}{c}
m \\
j
\end{array}\right)(-1)^{j} H_{n}^{(v)}(x-j) .
$$

By (1.5), we get

$$
\begin{aligned}
H B_{n}^{(v, k)}(x) & =e^{-\frac{v t^{2}}{2}} B_{n}^{(k)}(x)=\sum_{l=0}^{\infty} \frac{1}{l !}\left(-\frac{v}{2}\right)^{l} t^{2 l} B_{n}^{(k)}(x) \\
& =\sum_{l=0}^{\left[\frac{n}{2}\right]} \frac{1}{l !}\left(-\frac{v}{2}\right)^{l} \sum_{m=0}^{n} \frac{1}{(m+1)^{k}} \sum_{j=0}^{m}(-1)^{j}\left(\begin{array}{c}
m \\
j
\end{array}\right) t^{2 l}(x-j)^{n} \\
& =\sum_{l=0}^{\left[\frac{n}{2}\right]} \sum_{j=0}^{n}\left\{\sum_{m=j}^{n}\left(\begin{array}{c}
n \\
2 l
\end{array}\right) \frac{(2 l) !}{l !}\left(-\frac{v}{2}\right)^{l} \frac{(-1)^{j}\left(\begin{array}{c}
m \\
j
\end{array}\right)}{(m+1)^{k}}\right\}(x-j)^{n-2 l} .
\end{aligned}
$$

Therefore, by (2.7), we obtain the following theorem.

Theorem 3 For $n \geq 0$, we have

$$
H B_{n}^{(v, k)}(x)=\sum_{l=0}^{\left[\frac{n}{2}\right]} \sum_{j=0}^{n}\left\{\sum_{m=j}^{n}\left(\begin{array}{c}
n \\
2 l
\end{array}\right) \frac{(2 l) !}{l !}\left(-\frac{v}{2}\right)^{l} \frac{(-1)^{j}\left(\begin{array}{c}
m \\
j
\end{array}\right)}{(m+1)^{k}}\right\}(x-j)^{n-2 l} .
$$

By (2.6), we get

$$
\begin{aligned}
H B_{n}^{(v, k)}(x) & =\sum_{m=0}^{n} \frac{\left(1-e^{-t}\right)^{m}}{(m+1)^{k}} H_{n}^{(v)}(x) \\
& =\sum_{m=0}^{n} \frac{1}{(m+1)^{k}} \sum_{a=0}^{n-m} \frac{m !}{(a+m) !}(-1)^{a} S_{2}(a+m, m)(n)_{a+m} H_{n-a-m}^{(v)}(x) \\
& =\sum_{m=0}^{n} \sum_{a=0}^{n-m} \frac{(-1)^{n-a-m} m !}{(m+1)^{k}}\left(\begin{array}{c}
n \\
n-a
\end{array}\right) S_{2}(n-a, m) H_{a}^{(v)}(x) \\
& =(-1)^{n} \sum_{a=0}^{n}\left\{\sum_{m=0}^{n-a} \frac{(-1)^{m+a} m !}{(m+1)^{k}}\left(\begin{array}{l}
n \\
a
\end{array}\right) S_{2}(n-a, m)\right\} H_{a}^{(v)}(x),
\end{aligned}
$$

where $S_{2}(n, m)$ is the Stirling number of the second kind.

Therefore, by (2.8), we obtain the following theorem.

Theorem 4 For $n \geq 0$, we have

$$
H B_{n}^{(v, k)}(x)=(-1)^{n} \sum_{a=0}^{n}\left\{\sum_{m=0}^{n-a} \frac{(-1)^{a+m} m !}{(m+1)^{k}}\left(\begin{array}{l}
n \\
a
\end{array}\right) S_{2}(n-a, m)\right\} H_{a}^{(v)}(x) .
$$


From (1.19) and (2.1), we have

$$
\begin{aligned}
& H B_{n}^{(v, k)}(x)=\sum_{j=0}^{n}\left(\begin{array}{l}
n \\
j
\end{array}\right)\left\langle e^{-\frac{v t^{2}}{2}} \frac{\operatorname{Li}_{k}\left(1-e^{-t}\right)}{1-e^{-t}} \mid x^{n-j}\right\rangle x^{j} \\
& =\sum_{j=0}^{n}\left(\begin{array}{l}
n \\
j
\end{array}\right)\left\langle e^{-\frac{v t^{2}}{2}}\left|B_{n-j}^{(k)}(x)\right| x^{j}\right. \\
& =\sum_{j=0}^{n}\left(\begin{array}{l}
n \\
j
\end{array}\right) \sum_{l=0}^{\left[\frac{n-j}{2}\right]} \frac{\left(-\frac{v}{2}\right)^{l}}{l !}(n-j)_{2 l}\left\langle 1\left|B_{n-j-2 l}^{(k)}(x)\right| x^{j}\right. \\
& =\sum_{j=0}^{n}\left(\begin{array}{l}
n \\
j
\end{array}\right) \sum_{l=0}^{\left[\frac{n-j}{2}\right]} \frac{1}{l !}\left(-\frac{v}{2}\right)^{l}(n-j)_{2 l} B_{n-j-2 l}^{(k)} x^{j} \\
& =\sum_{j=0}^{n}\left\{\sum_{l=0}^{\left[\frac{n-j}{2}\right]}\left(\begin{array}{c}
n \\
j
\end{array}\right)\left(\begin{array}{c}
n-j \\
2 l
\end{array}\right) \frac{(2 l) !}{l !}\left(-\frac{v}{2}\right)^{l} B_{n-j-2 l}^{(k)}\right\} x^{j} .
\end{aligned}
$$

Therefore, by (2.9), we obtain the following theorem.

Theorem 5 For $n \geq 0$, we have

$$
H B_{n}^{(v, k)}(x)=\sum_{j=0}^{n}\left\{\sum_{l=0}^{\left[\frac{n-j}{2}\right]}\left(\begin{array}{c}
n \\
j
\end{array}\right)\left(\begin{array}{c}
n-j \\
2 l
\end{array}\right) \frac{(2 l) !}{l !}\left(-\frac{v}{2}\right)^{l} B_{n-j-2 l}^{(k)}\right\} x^{j}
$$

Remark By (1.17) and (2.1), we easily get

$$
H B_{n}^{(v, k)}(x+y)=\sum_{j=0}^{n}\left(\begin{array}{l}
n \\
j
\end{array}\right) H B_{j}^{(v, k)}(x) y^{n-j}
$$

We note that

$$
H B_{n}^{(v, k)}(x) \sim\left(g(t)=e^{\frac{v t^{2}}{2}} \frac{1-e^{-t}}{\operatorname{Li}_{k}\left(1-e^{-t}\right)}, f(t)=t\right) .
$$

From (1.18) and (2.11), we have

$$
H B_{n+1}^{(v, k)}(x)=\left(x-\frac{g^{\prime}(t)}{g(t)}\right) H B_{n}^{(v, k)}(x) .
$$

Now, we observe that

$$
\begin{aligned}
\frac{g^{\prime}(t)}{g(t)} & =(\log (g(t)))^{\prime} \\
& =\left(\log e^{\frac{v t^{2}}{2}}+\log \left(1-e^{-t}\right)-\log \left(\operatorname{Li}_{k}\left(1-e^{-t}\right)\right)\right)^{\prime} \\
& =v t+\frac{e^{-t}}{1-e^{-t}}\left(1-\frac{\operatorname{Li}_{k-1}\left(1-e^{-t}\right)}{\operatorname{Li}_{k}\left(1-e^{-t}\right)}\right) .
\end{aligned}
$$


By (2.12) and (2.13), we get

$$
\begin{aligned}
& H B_{n+1}^{(v, k)}(x) \\
& \quad=x H B_{n}^{(v, k)}(x)-\frac{g^{\prime}(t)}{g(t)} H B_{n}^{(v, k)}(x) \\
& \quad=x H B_{n}^{(v, k)}(x)-v n H B_{n-1}^{(v, k)}(x)-e^{-\frac{v t^{2}}{2}} \frac{t}{e^{t}-1} \frac{\mathrm{Li}_{k}\left(1-e^{-t}\right)-\mathrm{Li}_{k-1}\left(1-e^{-t}\right)}{t\left(1-e^{-t}\right)} x^{n} .
\end{aligned}
$$

It is easy to show that

$$
\begin{aligned}
\frac{\operatorname{Li}_{k}\left(1-e^{-t}\right)-\operatorname{Li}_{k-1}\left(1-e^{-t}\right)}{1-e^{-t}} & =\sum_{m=2}^{\infty}\left(\frac{1}{m^{k}}-\frac{1}{m^{k-1}}\right)\left(1-e^{-t}\right)^{m-1} \\
& =\left(\frac{1}{2^{k}}-\frac{1}{2^{k-1}}\right) t+\cdots .
\end{aligned}
$$

Thus, by (2.15), we get

$$
\frac{\mathrm{Li}_{k}\left(1-e^{-t}\right)-\mathrm{Li}_{k-1}\left(1-e^{-t}\right)}{t\left(1-e^{-t}\right)} x^{n}=\frac{\operatorname{Li}_{k}\left(1-e^{-t}\right)-\mathrm{Li}_{k-1}\left(1-e^{-t}\right)}{1-e^{-t}} \frac{x^{n+1}}{n+1}
$$

From (2.16), we can derive

$$
\begin{aligned}
& e^{-\frac{v t^{2}}{2}} \frac{t}{e^{t}-1} \frac{\mathrm{Li}_{k}\left(1-e^{-t}\right)-\mathrm{Li}_{k-1}\left(1-e^{-t}\right)}{t\left(1-e^{-t}\right)} x^{n} \\
& =\frac{1}{n+1}\left(\sum_{l=0}^{\infty} \frac{B_{l}}{l !} t^{l}\right)\left(H B_{n+1}^{(v, k)}(x)-H B_{n+1}^{(v, k-1)}(x)\right) \\
& =\frac{1}{n+1} \sum_{l=0}^{n+1} \frac{B_{l}}{l !} t^{l}\left(H B_{n+1}^{(v, k)}(x)-H B_{n+1}^{(v, k-1)}(x)\right) \\
& =\frac{1}{n+1} \sum_{l=0}^{n+1}\left(\begin{array}{c}
n+1 \\
l
\end{array}\right) B_{l}\left(H B_{n+1-l}^{(v, k)}(x)-H B_{n+1-l}^{(v, k-1)}(x)\right) .
\end{aligned}
$$

Therefore, by (2.14) and (2.17), we obtain the following theorem.

Theorem 6 For $n \geq 0$, we have

$$
\begin{aligned}
& H B_{n+1}^{(v, k)}(x) \\
& =x H B_{n}^{(v, k)}(x)-v n H B_{n-1}^{(v, k)}(x) \\
& \quad-\frac{1}{n+1} \sum_{l=0}^{n+1}\left(\begin{array}{c}
n+1 \\
l
\end{array}\right) B_{l}\left\{H B_{n+1-l}^{(v, k)}(x)-H B_{n+1-l}^{(v, k-1)}(x)\right\} .
\end{aligned}
$$

Let us take $t$ on the both sides of (2.18). Then we have

$$
\begin{aligned}
(n & +1) H B_{n}^{(v, k)}(x) \\
& =(x t+1) H B_{n}^{(v, k)}(x)-v n(n-1) H B_{n-2}^{(v, k)}(x)
\end{aligned}
$$




$$
\begin{aligned}
& -\frac{1}{n+1} \sum_{l=0}^{n+1}\left(\begin{array}{c}
n+1 \\
l
\end{array}\right)(n+1-l) B_{l}\left\{H B_{n-l}^{(v, k)}(x)-H B_{n-l}^{(v, k-1)}(x)\right\} \\
= & n x H B_{n-1}^{(v, k)}(x)+H B_{n}^{(v, k)}(x)-v n(n-1) H B_{n-2}^{(v, k)}(x) \\
& -\sum_{l=0}^{n}\left(\begin{array}{l}
n \\
l
\end{array}\right) B_{l}\left(H B_{n-l}^{(v, k)}(x)-H B_{n-l}^{(v, k-1)}(x)\right),
\end{aligned}
$$

where $n \geq 3$.

Thus, by (2.19), we obtain the following theorem.

Theorem 7 For $n \geq 3$, we have

$$
\begin{aligned}
\sum_{l=0}^{n}\left(\begin{array}{l}
n \\
l
\end{array}\right) B_{l} H B_{n-l}^{(v, k-1)}(x) \\
=(n+1) H B_{n}^{(v, k)}(x)-n\left(x+\frac{1}{2}\right) H B_{n-1}^{(v, k)}(x) \\
+n(n-1)\left(v+\frac{1}{12}\right) H B_{n-2}^{(v, k)}(x) \\
\quad+\sum_{l=0}^{n-3}\left(\begin{array}{l}
n \\
l
\end{array}\right) B_{n-l} H B_{l}^{(v, k)}(x) .
\end{aligned}
$$

By (1.5) and (1.8), we get

$$
\begin{aligned}
& H B_{n}^{(v, k)}(y) \\
& =\left\langle e^{-\frac{v t^{2}}{2}} \frac{\operatorname{Li}_{k}\left(1-e^{-t}\right)}{1-e^{-t}} e^{y t} \mid x^{n}\right\rangle \\
& =\left\langle\partial_{t}\left(e^{-\frac{v t^{2}}{2}} \frac{\mathrm{Li}_{k}\left(1-e^{-t}\right)}{1-e^{-t}} e^{y t}\right) \mid x^{n-1}\right\rangle \\
& =\left\langle\left(\partial_{t} e^{-\frac{v t^{2}}{2}}\right) \frac{\mathrm{Li}_{k}\left(1-e^{-t}\right)}{1-e^{-t}} e^{y t} \mid x^{n-1}\right\rangle \\
& +\left\langle e^{-\frac{v t^{2}}{2}}\left(\partial_{t} \frac{\operatorname{Li}_{k}\left(1-e^{-t}\right)}{1-e^{-t}}\right) e^{y t} \mid x^{n-1}\right\rangle \\
& +\left\langle e^{-\frac{v t^{2}}{2}} \frac{\operatorname{Li}_{k}\left(1-e^{-t}\right)}{1-e^{-t}}\left(\partial_{t} e^{y t}\right) \mid x^{n-1}\right\rangle \\
& =-v(n-1)\left\langle e^{-\frac{v t^{2}}{2}} \frac{\operatorname{Li}_{k}\left(1-e^{-t}\right)}{1-e^{-t}} e^{y t} \mid x^{n-2}\right\rangle \\
& +y\left\langle e^{-\frac{v t^{2}}{2}} \frac{\operatorname{Li}_{k}\left(1-e^{-t}\right)}{1-e^{-t}} e^{y t} \mid x^{n-1}\right\rangle \\
& +\left\langle e^{-\frac{v t^{2}}{2}}\left(\partial_{t} \frac{\operatorname{Li}_{k}\left(1-e^{-t}\right)}{1-e^{-t}}\right) e^{y t} \mid x^{n-1}\right\rangle \\
& =-v(n-1) H B_{n-2}^{(v, k)}(y)+y H B_{n-1}^{(v, k)}(y) \\
& +\left\langle e^{-\frac{v t^{2}}{2}}\left(\partial_{t} \frac{\mathrm{Li}_{k}\left(1-e^{-t}\right)}{1-e^{-t}}\right) e^{y t} \mid x^{n-1}\right\rangle .
\end{aligned}
$$


Now, we observe that

$$
\partial_{t}\left(\frac{\operatorname{Li}_{k}\left(1-e^{-t}\right)}{1-e^{-t}}\right)=\frac{\operatorname{Li}_{k-1}\left(1-e^{-t}\right)-\operatorname{Li}_{k}\left(1-e^{-t}\right)}{\left(1-e^{-t}\right)^{2}} e^{-t} .
$$

From (2.21), we have

$$
\begin{aligned}
& \left\langle e^{-\frac{v t^{2}}{2}}\left(\partial_{t} \frac{\mathrm{Li}_{k}\left(1-e^{-t}\right)}{1-e^{-t}}\right) e^{y t} \mid x^{n-1}\right\rangle \\
& =\left\langle e^{-\frac{v t^{2}}{2}}\left(\frac{\mathrm{Li}_{k-1}\left(1-e^{-t}\right)-\mathrm{Li}_{k}\left(1-e^{-t}\right)}{\left(1-e^{-t}\right)^{2}}\right) e^{-t} e^{y t} \mid \frac{1}{n} t x^{n}\right\rangle \\
& =\frac{1}{n}\left\langle e^{-\frac{v t^{2}}{2}} \frac{\mathrm{Li}_{k-1}\left(1-e^{-t}\right)-\mathrm{Li}_{k}\left(1-e^{-t}\right)}{1-e^{-t}} e^{y t} \mid \frac{t}{e^{t}-1} x^{n}\right\rangle \\
& =\frac{1}{n}\left\langle e^{-\frac{v t^{2}}{2}} \frac{\mathrm{Li}_{k-1}\left(1-e^{-t}\right)-\mathrm{Li}_{k}\left(1-e^{-t}\right)}{1-e^{-t}} e^{y t} \mid B_{n}(x)\right\rangle \\
& =\frac{1}{n} \sum_{l=0}^{n}\left(\begin{array}{l}
n \\
l
\end{array}\right) B_{l}\left\langle e^{-\frac{v t^{2}}{2}} \frac{\mathrm{Li}_{k-1}\left(1-e^{-t}\right)-\mathrm{Li}_{k}\left(1-e^{-t}\right)}{1-e^{-t}} e^{y t} \mid x^{n-l}\right\rangle \\
& =\frac{1}{n} \sum_{l=0}^{n}\left(\begin{array}{l}
n \\
l
\end{array}\right) B_{l}\left\{H B_{n-l}^{(v, k-1)}(y)-H B_{n-l}^{(v, k)}(y)\right\},
\end{aligned}
$$

where $B_{n}$ are the ordinary Bernoulli numbers which are defined by the generating function to be

$$
\frac{t}{e^{t}-1}=\sum_{n=0}^{\infty} \frac{B_{n}}{n !} t^{n}
$$

Therefore, by (2.20) and (2.22), we obtain the following theorem.

Theorem 8 For $n \geq 2$, we have

$$
\begin{aligned}
H B_{n}^{(v, k)}(x)= & -v(n-1) H B_{n-2}^{(v, k)}(x)+x H B_{n-1}^{(v, k)}(x) \\
& +\frac{1}{n} \sum_{l=0}^{n}\left(\begin{array}{l}
n \\
l
\end{array}\right) B_{l}\left(H B_{n-l}^{(v, k-1)}(x)-H B_{n-l}^{(v, k)}(x)\right) .
\end{aligned}
$$

Now, we compute

$$
\left\langle e^{-\frac{v t^{2}}{2}} \operatorname{Li}_{k}\left(1-e^{-t}\right) \mid x^{n+1}\right\rangle
$$

in two different ways.

On the one hand,

$$
\begin{aligned}
& \left\langle e^{-\frac{v t^{2}}{2}} \operatorname{Li}_{k}\left(1-e^{-t}\right) \mid x^{n+1}\right\rangle \\
& =\left\langle e^{-\frac{v t^{2}}{2}} \frac{\operatorname{Li}_{k}\left(1-e^{-t}\right)}{1-e^{-t}}\left(1-e^{-t}\right) \mid x^{n+1}\right\rangle \\
& =\left\langle e^{-\frac{v t^{2}}{2}} \frac{\operatorname{Li}_{k}\left(1-e^{-t}\right)}{1-e^{-t}} \mid\left(1-e^{-t}\right) x^{n+1}\right\rangle
\end{aligned}
$$


On the other hand,

$$
\begin{aligned}
\left\langle e^{-\frac{v t^{2}}{2}} \operatorname{Li}_{k}\left(1-e^{-t}\right) \mid x^{n+1}\right\rangle \\
=\left\langle\operatorname{Li}_{k}\left(1-e^{-t}\right) \mid e^{-\frac{v t^{2}}{2}} x^{n+1}\right\rangle \\
=\left\langle\int_{0}^{t}\left(\operatorname{Li}_{k}\left(1-e^{-s}\right)\right)^{\prime} d s \mid e^{-\frac{v t^{2}}{2}} x^{n+1}\right\rangle \\
=\left\langle\int_{0}^{t} e^{-s} \frac{\operatorname{Li}_{k-1}\left(1-e^{-s}\right)}{1-e^{-s}} d s \mid e^{-\frac{v t^{2}}{2}} x^{n+1}\right\rangle \\
=\left\langle\sum_{l=0}^{\infty}\left(\sum_{m=0}^{l}(-1)^{l-m}\left(\begin{array}{c}
l \\
m
\end{array}\right) B_{m}^{(k-1)} \frac{t^{l+1}}{(l+1) !}\right) \mid H_{n+1}^{(v)}(x)\right\rangle \\
=\sum_{l=0}^{n} \sum_{m=0}^{l}(-1)^{l-m}\left(\begin{array}{c}
l \\
m
\end{array}\right) B_{m}^{(k-1)} \frac{1}{(l+1) !}\left\langle t^{l+1} \mid H_{n+1}^{(v)}(x)\right\rangle \\
=\sum_{l=0}^{n} \sum_{m=0}^{l}(-1)^{l-m}\left(\begin{array}{c}
l \\
m
\end{array}\right)\left(\begin{array}{c}
n+1 \\
l+1
\end{array}\right) B_{m}^{(k-1)} H_{n-l}^{(v)} .
\end{aligned}
$$

Therefore, by (2.23) and (2.24), we obtain the following theorem.

Theorem 9 For $n \geq 0$, we have

$$
\begin{aligned}
& \sum_{m=0}^{n}(-1)^{n-m}\left(\begin{array}{c}
n+1 \\
m
\end{array}\right) H B_{m}^{(\nu, k)} \\
& \quad=\sum_{m=0}^{n} \sum_{l=m}^{n}(-1)^{l-m}\left(\begin{array}{c}
l \\
m
\end{array}\right)\left(\begin{array}{c}
n+1 \\
l+1
\end{array}\right) B_{m}^{(k-1)} H_{n-l}^{(v)} .
\end{aligned}
$$

Let us consider the following two Sheffer sequences:

$$
H B_{n}^{(v, k)}(x) \sim\left(e^{\frac{v t^{2}}{2}} \frac{1-e^{-t}}{\operatorname{Li}_{k}\left(1-e^{-t}\right)}, t\right)
$$

and

$$
\mathbb{B}_{n}^{(r)}(x) \sim\left(\left(\frac{e^{t}-1}{t}\right)^{r}, t\right) \quad\left(r \in \mathbb{Z}_{\geq 0}\right) .
$$

Let us assume that

$$
H B_{n}^{(v, k)}(x)=\sum_{m=0}^{n} C_{n, m} \mathbb{B}_{m}^{(r)}(x) .
$$


Then, by (1.20) and (1.21), we get

$$
\begin{aligned}
C_{n, m} & =\frac{1}{m !}\left\langle\left(\frac{e^{t}-1}{t}\right)^{r} t^{m} \mid e^{-\frac{v t^{2}}{2}} \frac{\operatorname{Li}_{k}\left(1-e^{-t}\right)}{1-e^{-t}} x^{n}\right\rangle \\
& =\frac{1}{m !}\left\langle\left(\frac{e^{t}-1}{t}\right)^{r} \mid t^{m} H B_{n}^{(v, k)}(x)\right\rangle=\frac{1}{m !}(n)_{m}\left\langle\left(\frac{e^{t}-1}{t}\right)^{r} \mid H B_{n-m}^{(v, k)}(x)\right\rangle \\
& =\left(\begin{array}{c}
n \\
m
\end{array}\right) \sum_{l=0}^{\infty} \frac{r !}{(l+r) !} S_{2}(l+r, r)\left\langle t^{l} \mid H B_{n-m}^{(v, k)}(x)\right\rangle \\
& =\left(\begin{array}{c}
n \\
m
\end{array}\right) \sum_{l=0}^{n-m}(n-m)_{l} \frac{r !}{(l+r) !} S_{2}(l+r, r) H B_{n-m-l}^{(v, k)} \\
& =\left(\begin{array}{c}
n \\
m
\end{array}\right) \sum_{l=0}^{n-m} \frac{\left(\begin{array}{c}
n-m \\
l
\end{array}\right)}{\left(\begin{array}{c}
l+r \\
r
\end{array}\right)} S_{2}(l+r, r) H B_{n-m-l}^{(v, k)} .
\end{aligned}
$$

Therefore, by (2.27) and (2.28), we obtain the following theorem.

Theorem 10 For $n, r \in \mathbb{Z}_{\geq 0}$, we have

$$
H B_{n}^{(v, k)}(x)=\sum_{m=0}^{n}\left\{\left(\begin{array}{c}
n \\
m
\end{array}\right) \sum_{l=0}^{n-m} \frac{\left(\begin{array}{c}
n-m \\
l
\end{array}\right)}{\left(\begin{array}{c}
l+r \\
r
\end{array}\right)} S_{2}(l+r, r) H B_{n-m-l}^{(v, k)}\right\} \mathbb{B}_{m}^{(r)}(x) .
$$

For $\lambda(\neq 1) \in \mathbb{C}, r \in \mathbb{Z}_{\geq 0}$, the Frobenius-Euler polynomials of order $r$ are defined by the generating function to be

$$
\left(\frac{1-\lambda}{e^{t}-\lambda}\right)^{r} e^{x t}=\sum_{n=0}^{\infty} H_{n}^{(r)}(x \mid \lambda) \frac{t^{n}}{n !} \quad(\text { see }[1,4,7,9,10]) .
$$

From (1.16) and (2.29), we note that

$$
H_{n}^{(r)}(x \mid \lambda) \sim\left(\left(\frac{e^{t}-\lambda}{1-\lambda}\right)^{r}, t\right) .
$$

Let us assume that

$$
H B_{n}^{(v, k)}(x)=\sum_{m=0}^{n} C_{n, m} H_{m}^{(r)}(x \mid \lambda)
$$

By (1.21), we get

$$
\begin{aligned}
C_{n, m} & =\frac{1}{m !}\left\langle\left(\frac{e^{t}-\lambda}{1-\lambda}\right)^{r} t^{m} \mid e^{-\frac{v t^{2}}{2}} \frac{\mathrm{Li}_{k}\left(1-e^{-t}\right)}{1-e^{-t}} x^{n}\right\rangle \\
& =\frac{(n)_{m}}{m !(1-\lambda)^{r}}\left\langle\sum_{l=0}^{r}\left(\begin{array}{l}
r \\
l
\end{array}\right)(-\lambda)^{r-l} e^{l t} \mid H B_{n-m}^{(v, k)}(x)\right\rangle \\
& =\left(\begin{array}{c}
n \\
m
\end{array}\right) \frac{1}{(1-\lambda)^{r}} \sum_{l=0}^{r}\left(\begin{array}{l}
r \\
l
\end{array}\right)(-\lambda)^{r-l}\left\langle 1 \mid e^{l t} H B_{n-m}^{(v, k)}(x)\right\rangle \\
& =\frac{\left(\begin{array}{c}
n \\
m
\end{array}\right)}{(1-\lambda)^{r}} \sum_{l=0}^{r}\left(\begin{array}{l}
r \\
l
\end{array}\right)(-\lambda)^{r-l} H B_{n-m}^{(v, k)}(l) .
\end{aligned}
$$


Therefore, by (2.31) and (2.32), we obtain the following theorem.

Theorem 11 For $n, r \in \mathbb{Z}_{\geq 0}$, we have

$$
H B_{n}^{(v, k)}(x)=\frac{1}{(1-\lambda)^{r}} \sum_{m=0}^{n}\left(\begin{array}{l}
n \\
m
\end{array}\right)\left\{\sum_{l=0}^{r}\left(\begin{array}{l}
r \\
l
\end{array}\right)(-\lambda)^{r-l} H B_{n-m}^{(\nu, k)}(l)\right\} H_{m}^{(r)}(x \mid \lambda) .
$$

\section{Competing interests}

The authors declare that they have no competing interests.

\section{Authors' contributions}

All authors contributed equally to the manuscript and typed, read, and approved the final manuscript.

\section{Author details}

'Department of Mathematics, Sogang University, Seoul, 121-742, Republic of Korea. ${ }^{2}$ Department of Mathematics, Kwangwoon University, Seoul, 139-701, Republic of Korea.

\section{Acknowledgements}

This work was supported by the National Research Foundation of Korea (NRF) grant funded by the Korea government (MOE) (No. 2012R1A1A2003786).

Received: 23 September 2013 Accepted: 7 November 2013 Published: 27 Nov 2013

\section{References}

1. Araci, S, Acikgoz, M: A note on the Frobenius-Euler numbers and polynomials associated with Bernstein polynomials. Adv. Stud. Contemp. Math. 22(3), 399-406 (2012)

2. Dere, R, Simsek, Y: Application of umbral algebra to some special polynomials. Adv. Stud. Contemp. Math. 22(3), 433-438 (2012)

3. Ding, D, Yang, J: Some identities related to the Apostol-Euler and Apostol-Bernoulli polynomials. Adv. Stud. Contemp. Math. 20(1), 7-21 (2010)

4. Ozden, H, Cangul, IN, Simsek, Y: Remarks on q-Bernoulli numbers associated with Daehee numbers. Adv. Stud. Contemp. Math. 18(1), 41-48 (2009)

5. Kaneko, M: Poly-Bernoulli numbers. J. Théor. Nr. Bordx. 9(1), 221-228 (1997)

6. Kim, DS, Kim, T, Dolgy, DV, Rim, SH: Some new identities of Bernoulli, Euler and Hermite polynomials arising from umbral calculus. Adv. Differ. Equ. 2013(2013), 73 (2013)

7. Kim, DS, Kim, T: Some identities of Frobenius-Euler polynomials arising from umbral calculus. Adv. Differ. Equ. 2012, $196(2012)$

8. Kim, DS, Kim, T, Lee, SH: A note on poly-Bernoulli polynomials arising from umbral calculus. Adv. Stud. Theor. Phys. 7(15), $731-744(2013)$

9. Kim, DS, Kim, T, Lee, SH: Poly-Cauchy numbers and polynomials with umbral calculus viewpoint. Int. J. Math. Anal. 7, 2235-2253 (2013)

10. Kim, DS, Kim, T, Lee, SH: Higher-order Cauchy of the first kind and poly-Cauchy of the first kind mixed type polynomials. Adv. Stud. Contemp. Math. 23, 543-554 (2013)

11. Kim, DS, Kim, T: Some identities of Bernoulli and Euler polynomials arising from umbral calculus. Adv. Stud. Contemp. Math. 23(1), 159-171 (2013)

12. Kurt, B, Simsek, Y: On Hermite based Genocchi polynomials. Adv. Stud. Contemp. Math. 23(1), 13-17 (2013)

13. Roman, S: The Umbral Calculus. Pure and Applied Mathematics, vol. 111. Academic Press, New York (1984). ISBN:0-12-594380-6

14. Roman, S, Rota, G-C: The umbral calculus. Adv. Math. 27(2), 95-188 (1978)

15. Rim, SH, Jeong, J: On the modified $q$-Euler numbers of higher order with weight. Adv. Stud. Contemp. Math. 22(1), 93-98 (2012)

16. Simsek, Y: Generating functions of the twisted Bernoulli numbers and polynomials associated with their interpolation functions. Adv. Stud. Contemp. Math. 16(2), 251-278 (2008)

10.1186/1687-1847-2013-343

Cite this article as: Kim and Kim: Hermite and poly-Bernoulli mixed-type polynomials. Advances in Difference Equations 2013, 2013:343 